\title{
Recent updates on phthalate exposure and human health: a special focus on liver toxicity and stem cell regeneration
}

\begin{abstract}
Phthalates have been blended in various compositions as plasticizers worldwide for a variety of purposes. Consequently, humans are exposed to a wide spectrum of phthalates that needs to be researched and understood correctly. The goal of this review is to focus on phthalate's internal exposure pathways and possible role of human digestion on liver toxicity. In addition, special focus was made on stem cell therapy in reverting liver toxicity. The known entry of higher molecular weight phthalates is through ingestion while inhalation and dermal pathways are for lower molecular weight phthalates. In human body, certain phthalates are digested through phase 1 (hydrolysis, oxidation) and phase 2 (conjugation) metabolic processes. The phthalates that are made bioavailable through digestion enter the blood stream and reach the liver for further detoxification, and these are excreted via urine and/or feces. Bis(2-ethylhexyl) phthalate (DEHP) is a compound well studied involving human metabolism. Liver plays a pivotal role in humans for detoxification of pollutants. Thus, continuous exposure to phthalates in humans may lead to inhibition of liver detoxifying enzymes and may result in liver dysfunction. The potential of stem cell therapy addressed herewith will revert liver dysfunction and lead to restoration of liver function properly.
\end{abstract}

Keyword: Biotransformation; Exposure; Human health; Liver toxicity; Metabolism; Phthalates; Stem cell therapy 